\title{
IDENTIFYING THE RISK FACTORS FOR LOW BONE MINERAL DENSITY DISORDERS IN HIV-POSITIVE PATIENTS UNDERGOING ANTIRETROVIRAL THERAPY FROM THE ROMANIAN COHORT
}

\author{
ANCA NEGRU ${ }^{1,2 \#}$, CĂTĂLIN TILIȘCAN ${ }^{1,2 *}$, ANA-MARIA TUDOR ${ }^{1,2 \#}$, CRISTIANA OPREA $^{1,3}$, \\ IRINA IANACHE ${ }^{3}$, MIHAI LAZĂR ${ }^{1,2 \#}$, DANIELA MUNTEANU ${ }^{1,2}$, MARIANA MARDARESCU ${ }^{2}$, \\ ADRIAN STREINU-CERCEL ${ }^{1,2}$, VICTORIA ARAMĂ ${ }^{1,2 \#}$, ȘTEFAN SORIN ARAMĂ $\breve{A}^{1,2}$
}

${ }^{I}$ Carol Davila University of Medicine and Pharmacy, Bucharest, Romania

${ }^{2}$ Prof. Dr. Matei Balș National Institute for Infectious Diseases, Bucharest, Romania

${ }^{3}$ Victor Babeș Clinical Hospital for Infectious and Tropical Diseases, Bucharest, Romania

*corresponding author: catalin.tiliscan@gmail.com

\# Authors with equal contribution.

\begin{abstract}
The aim of this study was to determine the prevalence and risk factors for osteopenia and osteoporosis among HIV infected patients from the Romanian cohort. We performed a cross-sectional study over a three-year period on HIV positive patients from the Romanian cohort which were in National Institute of Infectious Diseases "Prof. Dr. Matei Balş", Bucharest, Romania evidences. We calculated patient's body mass index (BMI), we determined CD4 cell count, HIV viral load and 25OH-vitamin D plasma levels. All patients had whole body bone mineral density (BMD) measured by Dual X-Ray Absorptiometry (DXA) scan and 29 of them also had hip and lumbar spine DXA scan. We enrolled 46 patients, 25 females. According to the $\mathrm{T}$ score at whole body examination 14 and 3 patients were diagnosed with osteopenia and osteoporosis, respectively. Lumbar spine osteopenia and osteoporosis were diagnosed in 7 and 2 patients, respectively. Hip osteopenia and osteoporosis were diagnosed in 4 and 2 patients, respectively. Regarding the $\mathrm{Z}$ score, 3 patients had values below the expected range at whole body examination and 2 had low values at both hip and lumbar spine. In patients with detectable HIV viral load low whole-body BMD was negatively correlated with HIV viremia. Patients with low plasma 25-OH-vitamin $\mathrm{D}$ levels had lower whole-body $\mathrm{Z}$ score means and those with viral hepatitis co-infections had lower lumber spine $\mathrm{T}$ and $\mathrm{Z}$ score means. High HIV viral load, low vitamin D levels and viral hepatitis co-infections may be indicators for BMD disturbances in patients with HIV infection.
\end{abstract}

\section{Rezumat}

Obiectivul studiului a fost determinarea prevalenței și a factorilor de risc pentru instalarea osteopeniei şi a osteoporozei la pacienții HIV pozitivi din cohorta românească. Am înrolat într-o perioadă de 3 ani pacienți infectați HIV din cohorta românească aflați în evidența Institutului Național de Boli Infecțioase „Prof. Dr. Matei Balș”, București, România. Am calculat indicele de masă corporală (IMC) şi am recoltat probe sangvine pentru a determina numărul celulelor CD4, viremia HIV și nivelul 25-OH-vitaminei D. Densitatea minerală osoasă (DMO) a fost evaluată prin Dual X-Ray Absorptiometry (DXA). Toți pacienții au efectuat whole body DXA, iar 29 au efectuat și DXA coloană lombară şi șold bilateral. Am înrolat 46 de pacienți dintre care 25 de femei. Conform scorului T la examinarea whole body 14 aveau osteopenie şi 3 osteoporoză, la examinarea coloanei lombare 7 aveau osteopenie și 2 osteoporoză. La examinarea șoldului 4 pacienți aveau osteopenie și 2 osteoporoză. Conform scorului Z la examinarea whole body, 3 pacienți au avut DMO scăzută, iar la examinarea şoldului și a coloanei lombare, 2 au avut DMO scăzută. La pacienții cu viremie detectabilă valoarea viremiei s-a corelat negativ cu DMO obținută la whole body DXA. Media scorurilor Z la examinarea whole body a fost mai scăzută la pacienții cu valori mici ale 25-OH-vitaminei $\mathrm{D}$, iar pacienții co-infectați cu virusuri hepatitice au avut media scorurilor $\mathrm{T}$ și $\mathrm{Z}$ de la coloana lombară mai scăzută. Încărcatura virală înaltă, nivelul scăzut al 25-OH-vitaminei D și co-infecția cu virusurile hepatitice sunt factori de risc pentru DMO scăzută la pacienții infectați HIV.

Keywords: HIV infection, bone mineral density, DXA

\section{Introduction}

After the emergence and appropriate use of antiretroviral treatment (ART) which led to increased survival of HIV-positive persons, HIV infection is no longer considered a fatal illness. Recent studies showed that the total life expectancy of a 20 -year- old seropositive patient increased from 30 years in 1996 , to 70 years in 2011 [1, 2]. For this reason, HIV infected patients are now at risk to develop different chronic non-AIDS related co-morbidities: cardiovascular diseases, diabetes mellitus, different types of malignancies, fragility fractures associated with low bone mineral density etc. Most of these 
co-morbidities are related to the ageing process that is accelerated in this category of patients [3-9].

Bone metabolism disturbances that lead to low bone mineral density (BMD) are common among HIV infected patients $[10,11]$. It is estimated that HIV positive individuals have a 6.7 -fold increased risk of low BMD and a 3.7-fold higher risk of osteopenia compared with uninfected subjects [12]. The ethyopathogesis of low BMD is multifactorial. In the case of HIV positive patients, the underlying mechanism of bone mineral density loss is still unclear. Besides general risk factors like smoking, sedentary lifestyle, alcohol and drug abuse, low body mass index, hypogonadism, 25-OH-vitamin D deficiency, glucocorticoid treatment this patients are also exposed to specific risk factors like chronic inflammation, immune disturbances and the presence of HIV virus that seem to have a negative impact on bone metabolism [13-15].

Regarding the epidemiology of HIV in Romania, in December 1990 the number of AIDS paediatric cases was higher than the total number of cases reported to the World Health Organization (WHO) from all the other countries from Europe. Between July 1989 and December 1990, 1090 paediatric AIDS cases were diagnosed, in $97 \%$ of the cases were involved children under 3-year-old, $67 \%$ of them being institutionalised children. It is estimated that more than 10,000 children were infected in this period with subtype F of HIV virus [16, 17]. These parenterally HIV-infected children form the Romanian paediatric cohort.

In June 2018, 15212 patients were registered in Romania with HIV infection - a significant proportion were young ( 5284 patients), aged between 25 and 29 years old, most of them belonging to the parenterally infected cohort. So we have to deal with a special kind of HIV patient: a young patient but with a long history of HIV infection and exposed to ART for a long time [16]. Therefore, co-morbidities associated with ageing and long-term exposure to ART, like fragility fractures, can have a high socio-economic impact for these patients, thus screening for osteopenia and osteoporosis may become mandatory even in young people.

The aim of this study was to evaluate the prevalence and risk factors for osteopenia and osteoporosis among HIV infected patients from the Romanian cohort, mainly diagnosed in early childhood and with long term ART exposure.

\section{Materials and Methods}

We performed a cross-sectional analysis on 46 consecutive HIV positive patients from Romania, enrolled over a three-year period. The Romanian HIV cohort includes patients diagnosed with HIV infection in early childhood and with long term of
ART exposure. The study took place in "Matei Balş" National Institute for Infectious Diseases from Bucharest, Romania, which is the largest infectious diseases centre in Romania and a reference centre for HIV infection management in Romania. The local ethics committee approved the study and all patients have signed an informed consent prior to study enrolment. HIV-positive patients from Romanian were included in the study, pregnant women were excluded.

We performed a detailed review of the medical records for each patient and we retrieved demographic data, information regarding the time of diagnosis, antiretroviral treatment (ART) history and adherence of treatment, current or past non- antiretroviral (ARV) medication. We also performed a complete physical check-up and we measured patient's weight and height to calculate the body mass index (BMI). Normal body weight was considered for a BMI between $18.5-24.99 \mathrm{~kg} / \mathrm{m}^{2}$, underweight for a BMI $\leq 18.5 \mathrm{~kg} / \mathrm{m}^{2}$ and overweight for a BMI $\geq 25 \mathrm{~kg} / \mathrm{m}^{2}$ (Table I) [18]. The plasma viral load was evaluated using Cobas ${ }^{\circledR}$ TaqMan $^{\circledR} 48$ system (Roche Diagnostics, Basel, Switzerland) with a detection limit of 20 copies $/ \mathrm{mL}$. The CD4 count was determined by flow cytometry using BD FACSCanto ${ }^{\circledR}$ II system.

25-OH-vitamin D serum levels were determined through electrochemiluminescence immunoassay method (ECLIA). Optimal vitamin D levels were considered above $30 \mathrm{ng} / \mathrm{mL}$, values between 10 and $29.9 \mathrm{ng} / \mathrm{mL}$ were considered suboptimal and vitamin D deficit was considered at values under $10 \mathrm{ng} / \mathrm{mL}$ [19]. All patients performed whole body and 29 (63\%) of them also had hip and lumbar spine dual energy Xray absorptiometry (DXA) using GE Lunar DPX-NT (General Electric Company, New-York, Connecticut, USA) scanner available in the radiology department. Bone mineral density (BMD) was expressed using T-score, which compares the subject's BMD with the average BMD of a young adult, and Z-score, which compares the subject's BMD with the BMD of a person with the same age, gender and race as the investigated patient.

We considered normal BMD when $\mathrm{T}$-score was higher than -1 . A T-score between -2.49 and -1 is diagnostic for osteopenia. Osteoporosis is considered in case of a T-score less than -2.5 or a Z-score less than -2 in postmenopausal women and in male patients aged at least 50 years old and measured in the lumbar spine, femoral neck or total hip. However, for female patients prior to menopause and in males younger than age 50 years, the World Health Organisation (WHO) recommends that the reporting of BMD should use Z-scores, not T-scores. A Zscore of -2.0 or higher was defined as "within the expected range for age", and a Z-score below -2.0 is "below the expected range for age". Furthermore, 
osteoporosis cannot be diagnosed in men under age 50 years using only the BMD value [20].

Statistical analysis

The collected data were analysed using the software $\mathrm{R}$, version 3.4.4 (The R Foundation for Statistical Computing, Vienna, Austria). For normal distributed variables, we expressed the mean \pm standard deviation (SD) and for non-normal distributions the median and interquartile range (IQR) were used. Visual inspection of histograms and Q-Q plots, the Shapiro-Wilk and Anderson-Darling tests were used to assess if the continuous variables were normally distributed. Linear correlations between normally distributed continuous variables were computed using the Pearson product moment correlation coefficient and Spearman's rankorder correlation was used to establish the strength and direction of the monotonic relationship between nonGaussian distributed variables. Mean comparison between normally distributed independent samples was performed using Student's T-test. The significance level was chosen to be 0.05 and all tests were twotailed.

\section{Results and Discussion}

46 HIV infected patients from the Romanian cohort were enrolled, 25 females (54.5\%) and 21 males $(45.7 \%)$, with a median age of 25 years $(24-26)$. Data regarding demographic characteristics, viral hepatitis co-infections and 25-OH-vitamin D levels are presented in Table I.

Table I

Demographic characteristics, hepatitis co-infection and 25-OH-vitamin D serum levels

\begin{tabular}{|c|c|c|}
\hline \multicolumn{2}{|l|}{ Age $^{1}$, years } & $25(24-26)$ \\
\hline \multicolumn{2}{|l|}{ Male gender ${ }^{2}$} & $21 / 45.7$ \\
\hline \multicolumn{2}{|l|}{ Smokers $^{2}$} & $12 / 26.1$ \\
\hline \multicolumn{2}{|l|}{$\mathrm{BMI}^{3}, \mathrm{~kg} / \mathrm{m}^{2}$} & $20.98 \pm 3.63$ \\
\hline \multirow{3}{*}{ Co-infections ${ }^{2}$} & $\mathrm{HBV}$ & $10 / 21.7$ \\
\hline & $\mathrm{HCV}$ & $3 / 6.5$ \\
\hline & $\mathrm{HBV}+\mathrm{HDV}$ & $1 / 2.2$ \\
\hline \multicolumn{2}{|l|}{ 25-OH-vitamin $\mathrm{D}^{3}, \mathrm{ng} / \mathrm{mL}$} & $22.80 \pm 10.77$ \\
\hline \multirow{3}{*}{ 25-OH-vitamin D level category ${ }^{2}$} & optimal & $11 / 23.9$ \\
\hline & suboptimal & $28 / 60.9$ \\
\hline & deficit & $4 / 8.7$ \\
\hline
\end{tabular}

BMI $=$ body mass index $;{ }^{1}$ Median (interquartile range) $;{ }^{2}$ Number $/$ percent $;{ }^{3}$ Mean \pm standard deviation

The median time from HIV diagnosis was 16 years (14 - 21). All the patients were under ART at the moment of examination for a median time of 15 years $(12.75$ - 16). In Table II are presented data regarding HIV infection and ARV treatment history.

Table II

Antiretroviral treatment history and characteristics

\begin{tabular}{|l|c|}
\hline Time from HIV diagnosis $^{1}$, years & $16(14-21)$ \\
\hline ART schemes $^{1}$ & $5(2.25-6)$ \\
\hline Time of exposure to ART $^{1}$ & $15(12.75-16)$ \\
\hline IP treatment $^{2}$ & $45 / 97.8$ \\
\hline IP exposure time, years $^{1}$ & $10(6-14)$ \\
\hline EFV treatment $^{2}$ & $24 / 52.17$ \\
\hline EFV exposure time, years $^{1}$ & $1.5(0-6.75)$ \\
\hline TDF treatment $^{2}$ & $2 / 4.3$ \\
\hline
\end{tabular}

${ }^{1}$ Median (interquartile range), ${ }^{2}$ Number/percent, ART $=$ antiretroviral treatment, $\mathrm{EFV}=$ efavirenz, IP = protease inhibitors, $\mathrm{TDF}=$ tenofovir disoproxil

At the time of DXA examination, undetectable HIV viral load was found in $26(56.5 \%)$ of the patients and median value of viral load was 0 copies $/ \mathrm{mL}$ (0 494.75). The median CD4 cell count was 523.5 cells/ $\mathrm{mm}^{3}\left(275.75-772.25 \mathrm{cell} / \mathrm{mm}^{3}\right)$ (Table III).

The mean whole-body BMD was $28.50 \pm 185.6$. The mean value for whole-body T-score was $-0.29 \pm 1.18$ and the mean value of Z-score was $0.19 \pm 1.05$. According to whole body T-score, 14 (30.4\%) patients had osteopenia and $3(6.5 \%)$ patients had osteoporosis. Two patients had BMD below the expected range according to $\mathrm{Z}$ score.

Table III

Distribution of studied cases by CD4 cell count and HIV viral load levels

\begin{tabular}{|c|c|c|}
\hline HIV plasma & Undetectable & $26 / 56.5$ \\
\cline { 2 - 3 } $\begin{array}{c}\text { viral load } \\
\text { (copies/mL) }\end{array}$ & $<200$ & $8 / 17.4$ \\
\cline { 2 - 3 } & $>200$ & $12 / 26.1$ \\
\hline \multirow{3}{*}{$\begin{array}{c}\text { CD4 cell count } \\
\text { and categories }^{\mathbf{1}}\end{array}$} & Median CD4 level & $\begin{array}{c}523.5 \\
(275.75-772.25)\end{array}$ \\
\cline { 2 - 3 } (cells/mm $^{3}$ ) & $>500$ & $24 / 52.2$ \\
\cline { 2 - 3 } & $200-499$ & $16 / 34.8$ \\
\cline { 2 - 3 } & $<200$ & $6 / 13$ \\
\hline
\end{tabular}

${ }^{1}$ Number / percent; ${ }^{2}$ Median (interquartile range)

Beside whole body DXA examination, 29 (63\%) patients also had hip and lumbar spine DXA examination. The mean BMD at hip and lumbar spine examination were $33.93 \pm 174.24$ and $1.12 \pm 0.11$, respectively. At hip examination the mean T-score value was $-0.25 \pm 1.13$ and mean $Z$-score value was $0 \pm 1.34$. According to T-score 4 patients $(13.8 \%)$ had osteopenia and 2 patients $(6.9 \%)$ had osteoporosis. Mean T-score and Z-score values at lumbar spine examination were $-0.55 \pm 1.03$ and $-0.19 \pm 1.05$, respectively. According to lumbar spine T-scores, 7 patients $(24.1 \%)$ had osteopenia and 2 patients $(6.9 \%)$ 
FARMACIA, 2019, Vol. 67, 3

had osteoporosis. Two patients (6.9\%) had Z-score values below the expected range (Tables IV, V).

Table IV

T-score results and interpretation

\begin{tabular}{|l|c|c|c|}
\hline & Whole body DXA (n= 46) & Hip DXA (n = 29) & Lumbar DXA (n = 29) \\
\hline Mean $^{\mathbf{1}}$ & $-0.29 \pm 1.18$ & $-0.25 \pm 1.13$ & $-0.55 \pm 1.03$ \\
\hline Osteopenia $^{2}$ & $14 / 30.4$ & $4 / 13.8$ & $7 / 24.1$ \\
\hline Osteoporosis $^{2}$ & $3 / 6.5$ & $2 / 6.9$ & $2 / 6.9$ \\
\hline
\end{tabular}

${ }^{1}$ Mean \pm standard deviation; ${ }^{2}$ Number/percent

Table V

Z-score results and interpretation

\begin{tabular}{|l|c|c|c|}
\hline & Whole body DXA $(\mathbf{n}=\mathbf{4 6})$ & Hip DXA $(\mathbf{n}=\mathbf{2 9})$ & Lumbar DXA (n= 29) \\
\hline Median $^{\mathbf{1}}$ & $0.19 \pm 1.05$ & $0 \pm 1.34$ & $-0.19 \pm 1.05$ \\
\hline Below the expected range $^{\mathbf{2}}$ & $3 / 6.5$ & $2 / 6.9$ & $2 / 6.9$ \\
\hline
\end{tabular}

${ }^{1}$ Mean \pm standard deviation; ${ }^{2}$ Number/percent

The percentage of patients with osteopenia at whole body DXA (30.4\%) was close to that of those with lumbar DXA (24.1\%). The lowest percentage of osteopenia was recorded at hip examination (13.8\%). Osteoporosis was diagnosed in similar proportions at all types of DXA $(6.5 \%$ at whole body DXA, $6.9 \%$ at both hip and lumbar spine DXA).

Lumbar osteopenia was diagnosed in almost twice more patients than hip osteopenia (4 patients vs. 7 patients) while the number of patients with osteoporosis was the same at both hip and lumbar DXA (2 patients). Same percentage of patients had BMD below the expected range at all types DXA examination.
There were no significant correlations between CD4 count, 25-OH-vitamin D levels, BMI, time from HIV diagnosis, ART exposure time, protease inhibitors (PI) exposure time or efavirenz (EFV) exposure time and the results of DXA examinations.

In patients with detectable HIV viral load (20 patients, $43.5 \%$ ), high plasma viral load during study period was associated with low values of whole-body BMD (rho $=-0.45, \mathrm{p}=0.04$ ) Whole body Z-score was significantly correlated to $25-\mathrm{OH}$-vitamin D values. Low 25-OH-vitamin D levels were associated with low Z-score results at whole body examination (rho = $0.30, p=0.04)$ (Tables VI, VII and VIII).

Table VI

Correlations between BMD and different variables

\begin{tabular}{|c|c|c|c|}
\hline & Whole body BMD & Hip BMD & Lumbar spine BMD \\
\hline CD4 count & rho $=0.03$ & rho $=-0.11$ & rho $=0.00$ \\
& $\mathrm{p}=0.83$ & $\mathrm{p}=0.56$ & $\mathrm{p}=0.95$ \\
\hline HIV viral load & $\mathbf{r h o}=\mathbf{- 0 . 4 5}$ & $\mathrm{rho}=-0.26$ & $\mathrm{rho}=-0.23$ \\
& $\mathbf{p}=\mathbf{0 . 0 4}$ & $\mathrm{p}=0.4$ & $\mathrm{p}=0.38$ \\
\hline BMI & $\mathrm{rho}=0.18$ & $\mathrm{rho}=-0.19$ & $\mathrm{rho}=-0.03$ \\
& $\mathrm{p}=0.21$ & $\mathrm{p}=0.32$ & $\mathrm{p}=0.85$ \\
\hline 25-OH-vitamin D levels & $\mathrm{PCC}=0.22$ & $\mathrm{PCC}=0.24$ & $\mathrm{PCC}=0.00$ \\
& $\mathrm{p}=0.15$ & $\mathrm{p}=0.23$ & $\mathrm{p}=0.98$ \\
\hline Time from HIV diagnosis & $\mathrm{rho}=0.14$ & $\mathrm{rho}=0.33$ & $\mathrm{rho}=0.20$ \\
& $\mathrm{p}=0.36$ & $\mathrm{p}=0.12$ & $\mathrm{p}=0.27$ \\
\hline ART exposure time & $\mathrm{rho}=-0.98$ & $\mathrm{rho}=0.24$ & $\mathrm{rho}=0.07$ \\
& $\mathrm{p}=0.51$ & $\mathrm{p}=0.21$ & $\mathrm{p}=0.65$ \\
\hline PI exposure time & $\mathrm{rho}=-0.08$ & $\mathrm{rho}=0.00$ & $\mathrm{rho}=-0.17$ \\
& $\mathrm{p}=0.60$ & $\mathrm{p}=0.98$ & $\mathrm{p}=0.32$ \\
\hline EFV exposure time & $\mathrm{rho}=0.14$ & $\mathrm{rho}=0.01$ & $\mathrm{rho}=0.11$ \\
& $\mathrm{p}=0.34$ & $\mathrm{p}=0.95$ & $\mathrm{p}=0.51$ \\
\hline
\end{tabular}

p-values were calculated with Mann-Whitney U test; BMI: body mass index, rho: Spearman`s rank correlation coefficient; PCC: Pearson correlation coefficient; ART: antiretroviral treatment; PI: protease inhibitors; EFV: efavirenz

Table VII

Correlations between T-scores and different variables

\begin{tabular}{|c|c|c|c|}
\hline & Whole body T-score & Hip T-score & Lumbar spine T-score \\
\hline CD4 count & rho $=0.08$ & rho $=0.06$ & rho $=0.12$ \\
& $p=0.57$ & $\mathrm{p}=0.72$ & $\mathrm{p}=0.52$ \\
\hline HIV viral load & $\mathrm{rho}=-0.4$ & $\mathrm{rho}=-0.24$ & $\mathrm{rho}=-0.2$ \\
& $\mathrm{p}=0.08$ & $\mathrm{p}=0.43$ & $\mathrm{p}=0.51$ \\
\hline BMI & $\mathrm{rho}=0.06$ & $\mathrm{rho}=-0.06$ & $\mathrm{rho}=0.03$ \\
& $\mathrm{p}=0.67$ & $\mathrm{p}=0.73$ & $\mathrm{p}=0.86$ \\
\hline 25-OH-vitamin D levels & $\mathrm{PCC}=0.18$ & $\mathrm{PCC}=0.35$ & $\mathrm{PCC}=0.08$ \\
& $\mathrm{p}=0.23$ & $\mathrm{p}=0.86$ & $\mathrm{p}=0.67$ \\
\hline
\end{tabular}


FARMACIA, 2019, Vol. 67, 3

\begin{tabular}{|c|c|c|c|}
\hline & Whole body T-score & Hip T-score & Lumbar spine T-score \\
\hline Time from HIV diagnosis & $\mathrm{rho}=0.11$ & $\mathrm{rho}=0.05$ & $\mathrm{rho}=0.14$ \\
& $\mathrm{p}=0.49$ & $\mathrm{p}=0.81$ & $\mathrm{p}=0.49$ \\
\hline ART exposure time & $\mathrm{rho}=0.05$ & $\mathrm{rho}=0.04$ & $\mathrm{rho}=0.03$ \\
& $\mathrm{p}=0.97$ & $\mathrm{p}=0.80$ & $\mathrm{p}=0.86$ \\
\hline PI exposure time & $\mathrm{rho}=-0.22$ & $\mathrm{rho}=-0.14$ & $\mathrm{rho}=-0.27$ \\
& $\mathrm{p}=0.14$ & $\mathrm{p}=0.46$ & $\mathrm{p}=0.16$ \\
\hline EFV exposure time & $\mathrm{rho}=0.19$ & $\mathrm{rho}=0.02$ & $\mathrm{rho}=0.04$ \\
& $\mathrm{p}=0.20$ & $\mathrm{p}=0.91$ & $\mathrm{p}=0.80$ \\
\hline
\end{tabular}

p-values were calculated with Mann-Whitney U test; BMI: body mass index, rho: Spearman`s rank correlation coefficient; PCC: Pearson correlation coefficient; ART: antiretroviral treatment; PI: protease inhibitors; EFV: efavirenz

Table VIII

Correlations between Z-scores and different variables

\begin{tabular}{|c|c|c|c|}
\hline & Whole body Z-score & Hip Z-score & Lumbar spine Z-score \\
\hline CD4 count & rho $=0.06$ & rho $=-0.04$ & rho $=0.17$ \\
& $\mathrm{p}=0.65$ & $\mathrm{p}=0.82$ & $\mathrm{p}=0.36$ \\
\hline HIV viral load & $\mathrm{rho}=-0.29$ & $\mathrm{rho}=-0.22$ & $\mathrm{rho}=-0.07$ \\
& $\mathrm{p}=0.20$ & $\mathrm{p}=0.48$ & $\mathrm{p}=0.81$ \\
\hline BMI & $\mathrm{rho}=-0.14$ & $\mathrm{rho}=-0.21$ & $\mathrm{rho}=-0.13$ \\
& $\mathrm{p}=0.34$ & $\mathrm{p}=0.26$ & $\mathrm{p}=0.48$ \\
\hline 25-OH-vitamin D levels & $\mathbf{P C C}=\mathbf{0 . 3 0}$ & $\mathrm{PCC}=0.17$ & $\mathrm{PCC}=0.02$ \\
& $\mathbf{p}=\mathbf{0 . 0 4}$ & $\mathrm{p}=0.39$ & $\mathrm{p}=0.89$ \\
\hline Time from HIV diagnosis & $\mathrm{rho}=0.04$ & $\mathrm{rho}=-0.17$ & $\mathrm{rho}=0.01$ \\
& $\mathrm{p}=0.77$ & $\mathrm{p}=0.42$ & $\mathrm{p}=0.94$ \\
\hline ART exposure time & $\mathrm{rho}=-0.05$ & $\mathrm{rho}=0.08$ & $\mathrm{rho}=0.01$ \\
& $\mathrm{p}=0.97$ & $\mathrm{p}=0.65$ & $\mathrm{p}=0.94$ \\
\hline PI exposure time & $\mathrm{rho}=-0.09$ & $\mathrm{rho}=-0.02$ & $\mathrm{rho}=-0.20$ \\
& $\mathrm{p}=0.52$ & $\mathrm{p}=0.89$ & $\mathrm{p}=0.30$ \\
\hline EFV exposure time & $\mathrm{rho}=0.06$ & $\mathrm{rho}=-0.03$ & $\mathrm{rho}=-0.02$ \\
& $\mathrm{p}=0.66$ & $\mathrm{p}=0.87$ & $\mathrm{p}=0.89$ \\
\hline
\end{tabular}

p-values were calculated with Mann-Whitney U test;BMI=body mass index, rho: Spearman`s rank correlation coefficient; PCC: Pearson correlation coefficient; ART: antiretroviral treatment; PI: protease inhibitors; EFV: efavirenz

We assessed the following potential factors associated with lower bone mineral density: female gender, smoking status, vitamin D levels and EFV exposure (Table IX).

No significant differences between the mean wholebody T-score $(p=0.14)$ and Z-score $(0.27)$, hip Tscore $(0.63)$ and $Z$-score $(p=0.49)$, lumbar spine $T$ score $(p=0.11)$ and $Z$-score $(p=0.11)$ were found in male versus female patients.

There were no significant differences between the mean whole-body T-score $(p=0.23)$ and Z-score $(p=0.25)$, hip T-score $(p=0.058)$ and Z-score $(p=0.092)$ or lumbar spine T-score $(p=0.4)$ and $Z$-score $(p=0.74)$ for smokers versus non-smokers patients. Patients with viral hepatitis co-infection had significantly lower mean lumbar spine T-score $(p=0.041)$ and Z-score $(p=0.037)$ than patients without hepatitis co-infection.

The mean whole-body Z-score was significantly higher in optimal 25-OH-vitamin D levels patients versus low 25-OH-vitamin D group patients $(\mathrm{p}=0.029)$. In low 25-OH-vitamin D group patients, we included patients with suboptimal levels of 25-OH-vitamin $\mathrm{D}$ and patients with 25-OH-vitamin D deficit.

We found no significant differences between the mean whole-body T-score $(p=0.15)$ and Z-score $(p=-0.49)$, hip T-score $(p=0.84)$ and Z-score $(p=0.71)$ and lumbar spine T-score $(p=0.57)$ and $Z$-score $(p=0.91)$ for patients treated with EFV versus patients never exposed to EFV.

Table IX

Comparison of DXA results between different subgroups of study patients

\begin{tabular}{|c|c|c|c|c|c|c|}
\hline & $\begin{array}{c}\text { Whole body } T \text { - } \\
\text { score }\end{array}$ & $\begin{array}{c}\text { Whole body } Z \text { - } \\
\text { score }\end{array}$ & Hip T-score & Hip Z-score & \begin{tabular}{|c|}
$\begin{array}{c}\text { Lumbar spine } \mathrm{T}- \\
\text { score }\end{array}$ \\
\end{tabular} & \begin{tabular}{|c}
$\begin{array}{c}\text { Lumbar spine Z- } \\
\text { score }\end{array}$ \\
\end{tabular} \\
\hline Male vs. $f$ & $\begin{array}{c}\mathrm{p}=0.14 \\
\text { CI } 95 \%=-1.22- \\
0.181\end{array}$ & $\begin{array}{c}\mathrm{p}=0.27 \\
\text { CI } 95 \%=-0.98- \\
0.28\end{array}$ & $\begin{array}{c}\mathrm{p}=0.63 \\
\text { CI 95\% }=-1.06- \\
0.65\end{array}$ & $\begin{array}{c}\mathrm{p}=0.49 \\
\text { CI 95\% }=-1.29- \\
0.64\end{array}$ & \begin{tabular}{|c|}
$\mathrm{p}=0.11$ \\
CI $95 \%=-1.39-$ \\
0.15 \\
\end{tabular} & $\begin{array}{c}\mathrm{p}=0.11 \\
\text { CI } 95 \%=-1.41- \\
0.15\end{array}$ \\
\hline $\begin{array}{l}\text { Smokers vs. non- } \\
\text { smokers }\end{array}$ & $\begin{array}{c}\mathrm{p}=0.24 \\
\text { CI } 95 \%=-1.29- \\
0.34\end{array}$ & $\begin{array}{c}\mathrm{p}=0.25 \\
\text { CI } 95 \%=-1.15- \\
0.31\end{array}$ & $\begin{array}{c}\mathrm{p}=0.058 \\
\text { CI } 95 \%=-1.92- \\
0.04\end{array}$ & $\begin{array}{c}\mathrm{p}=0.092 \\
\text { CI } 95 \%=-1.85- \\
0.16\end{array}$ & $\begin{array}{c}\mathrm{p}=0.4 \\
\text { CI } 95 \%=-0.8- \\
0.33\end{array}$ & $\begin{array}{c}\mathrm{p}=0.747 \\
\text { CI } 95 \%=-0.85- \\
0.62\end{array}$ \\
\hline $\begin{array}{l}\text { Hepatitis co-infection } \\
\text { vs. no hepatitis co- } \\
\text { infection }\end{array}$ & $\begin{array}{c}\mathrm{p}=0.112 \\
\text { CI } 95 \%=-0.16- \\
1.48\end{array}$ & $\begin{array}{c}\mathrm{p}=0.138 \\
\text { CI } 95 \%=-0.19- \\
1.33\end{array}$ & $\begin{array}{c}\mathrm{p}=0.068 \\
\text { CI } 95 \%=-0.08- \\
2.01\end{array}$ & $\begin{array}{c}\mathrm{p}=0.12 \\
\text { CI 95\% }=-0.24- \\
1.92\end{array}$ & \begin{tabular}{|c|}
$p=0.041$ \\
CI $95 \%=0.04-$ \\
1.98
\end{tabular} & $\begin{array}{c}p=0.037 \\
\text { CI } 95 \%=0.06- \\
1.87\end{array}$ \\
\hline
\end{tabular}


FARMACIA, 2019, Vol. 67, 3

\begin{tabular}{|c|c|c|c|c|c|c|}
\hline & $\begin{array}{c}\text { Whole body T- } \\
\text { score }\end{array}$ & $\begin{array}{c}\text { Whole body Z- } \\
\text { score }\end{array}$ & "Hip T-score & "Hip Z-score & \begin{tabular}{|c|}
$\begin{array}{c}\text { Lumbar spine } \mathrm{T}- \\
\text { score }\end{array}$ \\
\end{tabular} & \begin{tabular}{|c|}
$\begin{array}{c}\text { Lumbar spine } \mathrm{Z}- \\
\text { score }\end{array}$ \\
\end{tabular} \\
\hline $\begin{array}{l}\text { Optimal vs. low 25- } \\
\text { OH-vitamin D levels }\end{array}$ & $\begin{array}{c}\mathrm{p}=0.181 \\
\text { CI } 95 \%=-0.31- \\
1.54\end{array}$ & $\begin{array}{c}p=0.029 \\
\text { CI } 95 \%=0.10- \\
1.72\end{array}$ & $\begin{array}{c}\mathrm{p}=0.9 \\
\text { CI } 95 \%=-0.89- \\
0.79\end{array}$ & $\begin{array}{c}\mathrm{p}=0.94 \\
\text { CI } 95 \%=-1.07- \\
0.99\end{array}$ & \begin{tabular}{|c|}
$\mathrm{p}=0.68$ \\
CI $95 \%=-0.54-$ \\
0.80
\end{tabular} & $\begin{array}{c}\mathrm{p}=0.58 \\
\text { CI 95\% }=-0.64- \\
1.13\end{array}$ \\
\hline EFV exposure & $\begin{array}{c}\mathrm{p}=0.15 \\
\text { CI } 95 \%=-1.23- \\
0.2\end{array}$ & $\begin{array}{c}\mathrm{p}=-0.49 \\
\text { CI } 95 \%=-0.9- \\
0.44\end{array}$ & $\begin{array}{c}\mathrm{p}=0.84 \\
\text { CI } 95 \%=-1-0.82\end{array}$ & $\begin{array}{c}\mathrm{p}=0.71 \\
\text { CI } 95 \%=-0.96- \\
1.36\end{array}$ & $\begin{array}{c}\mathrm{p}=0.57 \\
\text { CI } 95 \%=-1.09- \\
0.62\end{array}$ & $\begin{array}{c}\mathrm{p}=0.91 \\
\text { CI 95\% }=-0.93- \\
0.84\end{array}$ \\
\hline
\end{tabular}

p-values were calculated with Mann-Whitney $U$ test; $C I=95 \%$ confidence interval; $E F V=$ efavirenz

The cause for low BMD in HIV infected patients is still unknown, but antiretroviral therapy, hypovitaminosis D and HIV infection itself, in addition to conventional risk factors (smoking, low BMI, physical inactivity, hypogonadism) seem to have an important role in BMD disturbances [21].

In contrast to other studies regarding bone mineral density abnormalities in HIV infected patients, our study included only young patients infected with HIV in childhood, with multiple ARV regimens (patients from the Romania). These patients represent an important part of all the people living with HIV from our country - more than 5000 patients from a total of 15212 patients alive at the middle of 2018 , requiring a special medical approach [22]. Comorbidities associated with the process of ageing, diagnosed earlier in life for this category of patients, can have dramatic consequences. Fragility fractures represent the most feared complication of bone metabolism disorders. As HIV infection is now considered a chronic disease and life expectancy of HIV positive patients continues to increase, prevention of bone mineral density abnormalities may become an important therapeutic goal.

Compared with other studies from medical literature, we revealed a low prevalence of abnormal bone density: at whole body examination $17(36.9 \%)$ patients had low mineral density out of which 14 (30.4\%) were diagnosed with osteopenia and $3(6.5 \%)$ of them with osteoporosis. Out of $29(63 \%)$ patients that also had hip and lumbar spine DXA, 4 (13.8\%) patients had osteopenia and $2(6.9 \%)$ osteoporosis in the hip region. Seven patients $(24.1 \%)$ had lumbar spine osteopenia and 2 patients $(6.9 \%)$ had lumbar spine osteoporosis, respectively. An explanation could be that the median age of our patients ( 25 years) is significantly lower than of those included in other studies.

Patients with viral hepatitis co-infections and with low 25-OH-vitamin D levels had statistically significant lower lumber spine $\mathrm{T}$ and $\mathrm{Z}$-scores and whole-body Z-score, respectively.

A recent analysis from Spain which included 107 HIV positive patients with a median age of 46.5 (43 $51.5)$ years old showed a higher prevalence of low bone mineral density (63 patients, 58.9\%), 11 (10.9\%) of them being diagnosed with osteoporosis and 52 (48\%) with osteopenia. The authors also found a higher number of patients with low serum levels of
25-OH-vitamin D: 86 patients (89.4\%). In this study viral hepatitis $\mathrm{C}$ co-infection was associated with low BMD in the hip region, while in our study, hepatitis $\mathrm{B}$ and $\mathrm{C}$ viral co-infection was associated with lumbar spine reduced BMD. They also found significant correlations between hip bone mineral density and male gender, active smoking, low BMI, prolonged period between HIV diagnosis and initiation of ART, exposure to PI and methadone. Low lumbar spine BMD was associated with low CD4 cell count [23].

We found no correlation between DXA results and time from HIV diagnosis, CD4 cell count, BMI, smoking or exposure to EFV or IP. As we mentioned before, it is very probable that TDF was not involved in BMD disturbances in this case due to the fact that only 2 patients were under treatment this drug, all of them being exposed less than a year to TDF at the moment DXA was performed. None of our patients was under treatment with methadone.

Although in our study BMI was not associated with $\mathrm{BMD}$, low BMI is a risk factor for low bone mineral density in both HIV positive and HIV negative patients [23, 24]. A study on 918 HIV infected patients showed that T-scores at femoral neck and lumbar spine were positively correlated with BMI $(\mathrm{p}<0.001)$ and that underweight patients had a higher risk of osteopenia and osteoporosis [25]. In our study we found no correlation between BMD and BM probably due to relatively small number of patients included.

Low levels of 25-OH-vitamin D are associated with a higher risk to develop osteopenia or osteoporosis in both HIV positive and HIV negative patients. Many studies demonstrated that HIV positive population is more likely to have 25-OH-vitamin D deficiency in contrast to HIV negative subjects. Besides classic risk factors like reduced exposure to light, low vitamin D intake diet, female sex, high BMI, hyperparathyroidism, there may be some HIV- related factors for vitamin D levels disturbances [26, 27]. The mechanism responsible for low vitamin D levels in HIV infected subjects is not clear. Chronic inflammation may have a role due to high levels of TNF $\alpha$ which determine renal $1 \alpha$-hydroxylase impairment, reducing parathyroid hormone's (PTH) stimulatory effect on producing the active form of vitamin D (1,25-OH-Vitamin D).

HIV infection itself was described as a risk factor for this abnormality [28, 29]. Recently, some experimental 
studies discovered that ARV drugs, especially protease inhibitors and non-nucleoside reverse transcriptase inhibitors, could also be associated with vitamin D disturbances [30-32]. It was shown that ritonavir inhibits the production of $1,25(\mathrm{OH})$ vitamin $\mathrm{D}$ in the kidneys, while efavirenz is responsible for $25(\mathrm{OH})-$ vitamin D hydrolyzation to its inactive form 24,25 $(\mathrm{OH})_{2}$ vitamin D [33].

In our study $69.6 \%$ of the patients had low levels of 25-OH-vitamin D. This prevalence is similar to those reported in other studies [33, 34]. We also found that patients with low 25-OH-vitamin D levels had lower Z-score at whole body DXA. Atteritao et al. in a case control study showed that vertebral fractures were associated with vitamin D insufficiency [35]. Chronic inflammation syndrome present in all HIV positive patients regardless of the efficacy of the ARV treatment, but more pronounced in patient with treatment failure, is responsible for bone metabolism disturbances. Tumour necrosis factor $\alpha$ (TNF $\alpha$ ) and interleukin-6 (IL-6) seem to be responsible for osteoclastogenesis and increased bone turnover [36-38]. In a study from "Matei Balş" Institute of Bucharest, Romania, the authors did not find any correlations between inflammation markers and BMD, despite the fact that systemic inflammation was found in a large proportion of patients [17].

In our study, in case of patients with detectable HIV viral loads, we found a negative correlation between viral load and whole-body BMD. Low BMD was more frequent diagnosed in patients with high viral loads. An explanation for this correlation may be the magnitude of the inflammatory response which is more pronounced in patients with viral failure. This finding needs further follow-up because at the moment there are not many studies showing similar results. A study from Turkey on 126 HIV seropositive patients also found statistically association between HIV viral load at diagnosis and decreased BMD [39]. Among ARV drugs, TDF and protease inhibitors were associated with bone demineralisation. TDF nephrotoxic effects are responsible for high PTH levels secondary to hypophosphatemia caused by proximal tubules epithelial cell damage, leading to increased bone resorption. Low levels of 1,25-OHvitamin $\mathrm{D}$ are also the result of renal impairment in patients undergoing ARV schemes including TDF which decreases the rate of alpha-hydroxylation of 25-OH-vitamin D [40]. Many studies that compared TDF containing ART regimens with non-TDF ART schemes observed that bone turnover was accelerated in patients treated with TDF [41, 42]. In our study group, it is less likely that TDF influenced BMD since TDF was available in Romania for less than a year when our study started (TDF is available in Romania form January 2014).

PIs are also associated with low BMD. The pathogenic mechanisms that lead to bone metabolism disturbances are related to vitamin D and PTH metabolism disorders, stimulation of osteoclast resorption and inhibition of osteoblasts functions [43]. Almost all our patients were exposed to protease inhibitors, this class of drugs being one of the first ART classes discovered and used. In our study 54 patients $(98.2 \%)$ had IP in their ART schemes for a median time of 10 years (7 13). We found no correlation between exposure time to PI and BMD.

EFV is another ARV drug that can cause low BMD. Some studies revealed that this drug is responsible for low 25-OH-vitamin D levels, but the mechanisms through which EFV interfere with vitamin D are not clear [43]. In our study, thirty patients $(54.54 \%)$ had treatment regimens containing EFV for a median time of 2 years $(0.0-5.5)$, but there was no correlation between exposure or exposure time to EFV and BMD. While in large studies EFV treatment was associated with BMD disturbances, an explication why in our study we found no correlation between this two is that the exposure time to this drug was relatively short. The strength of our study is represented by the included patients which are representative for HIV infected population form Romania - young adults with a median age of 25 years $(24-26)$, parenterally infected in their childhood with a median time from diagnosis of 17 years (15 - 21), with a long exposure to ARV drugs (median exposure 15 years $(13-18)$ ) and a high number of ARV schemes (median treatment schemes 5 schemes $(3-6))$. Data regarding this type of patients are limited in international medical literature.

The weak point of this study is represented by the small number of patients included and heterogeneity of the ARV treatment schemes, which makes it difficult to analyse the impact of each type of drug on BMD. Most of the patients had numerous ARV regimens which included almost all therapeutic classes of drugs. Even in this situation we can definitely assume that TDF has not played a role in BMD reduction because this drug became available in Romania at the beginning of 2014.

\section{Conclusions}

We report that a quarter of our young HIV infected patients, with a long history of the infection and many ARV treatment schemes, had abnormal bone mineral density. Although this percentage is not as high as reported in other studies, it is significant taking into consideration the young age of the included patients. High viral load was the main risk factor for osteopenia and osteoporosis. As we did not find correlations between the time of ART or type of antiretroviral drugs exposure, but high viral load was associated with low BMD, we suppose that the effect of virological failure on BMD disturbances may be more important than ART toxicity in young HIV infected patients. This aspect is very important because undetected 
FARMACIA, 2019, Vol. 67, 3

osteoporosis can lead to fragility fractures, especially in active patients, leading to high morbidity and alteration of socio-economic integration. DXA examination may become mandatory for patients with virological failure, low levels of 25-OH-vitamin D and viral hepatitis co-infection.

\section{Acknowledgement}

The whole medical staff of "Matei Balș" National Institute of Infectious Diseases, who diagnosed, treated and followed up the HIV infected patients from the Romanian cohort; to HIV positive patients who accepted to participate to this study; to the medical staff from Radiology Department of "Matei Balș” Institute.

\section{References}

1. Castronuovo D, Cacopardo B, Pinzone MR, Di Rosa M, Martellotta F, Schioppa O, Moreno S, Nunnari G, Bone disease in the setting of HIV infection: update and review of the literature. Eur Rev Med Pharmacol Sci., 2013; 17(18): 2413-2419.

2. Marcus JL, Chao CR, Leyden WA, Xu L, Klein DB, Towner WJ, Horberg MA, Silverberg MJ, Narrowing the gap in life expectancy between HIV-infected and HIV-uninfected individuals with access to care. J Acquir Immune Defic Syndr., 2016; 73(1): 39-46.

3. Zanet E, Berretta M, Martellotta F, Cacopardo B, Tavio M, Berretta S, Tirelli U, Anal cancer: Focus on HIV-positive patients in the HAART-era. Curr HIV Res., 2011; 9(2): 70-81.

4. Spina M, Carbone A, Gloghini A, Serraino D, Berretta M, Tirelli U, Hodgkin's Disease in Patients with HIV Infection. Adv Hematol., 2011; 2011: 1-7.

5. Nunnari G, Berretta M, Pinzone MR, Di Rosa M, Berretta S, Cunsolo G, Malaguarnera M, Cosentino S, De Paoli P, Schnell JM, Cacopardo B, Hepatocellular carcinoma in HIV positive patients. Eur Rev Med Pharmacol Sci., 2012; 16(9): 1257-1270.

6. Monroe AK, Glesby MJ, Brown TT, Diagnosing and managing diabetes in HIV-infected patients: current concepts. Clin Infect Dis., 2015; 60(3): 453-462.

7. Freiberg MS, So-Armah K, HIV and cardiovascular disease: We Need a mechanism, and we need a plan. J Am Heart Assoc., 2016; 4(3): 1-3.

8. Rubinstein PG, Aboulafia DM, Zloza A, Malignancies in HIV/AIDS: From epidemiology to therapeutic challenges. AIDS, 2014; 28(4): 453-465.

9. Walker Harris V, Brown TT, Bone loss in the HIVinfected patient: evidence, clinical implications, and treatment strategies. J Infect Dis., 2012; 205(Suppl 3): S391-398.

10. Moore AL, Vashisht A, Sabin CA, Mocroft A, Madge S, Phillips AN, Studd JW, Johnson MA, Reduced bone mineral density in HIV-positive individuals. AIDS, 2001; 15(13): 1731-1733.

11. Tebas P, Powderly WG, Claxton S, Marin D, Tantisiriwat W, Teitelbaum SL, Yarasheski KE, Accelerated bone mineral loss in HIV-infected patients receiving potent antiretroviral therapy. AIDS, 2000; 14(4): F63-67.
12. Brown TT, Qaqish RB, Antiretroviral therapy and the prevalence of osteopenia and osteoporosis: a meta-analytic review. AIDS, 2006; 20(17): 2165-2174.

13. Huangfu Q, Li M, Xiao L, Tao H, Wang W, Fei X, Effect of inhaled glucocorticoids on chronic obstructive pulmonary disease in male patients with osteoporosis. Farmacia, 2018; 66(1): 70-77.

14. Rothman MS, Bessesen MT, HIV infection and osteoporosis: pathophysiology, diagnosis, and treatment options. Curr Osteoporos Rep., 2012; 10(4): 270-277.

15. Güerri-Fernández R, Villar-García J, Díez-Pérez A, Prieto-Alhambra D, HIV infection, bone metabolism, and fractures. Arq Bras Endocrinol Metabol., 2014; 58(5): 478-483.

16. National Commission for Fighting Anti-AIDS Infectious Disease Institute "Prof. Dr. Matei Bals", available in Romanian, at: http://cnlas.ro.

17. Munteanu D, Negru A, Mihăilescu R, Tilișcan C, Tudor AM, Lazăr M, Aramă ȘS, Ion D, Popescu C, Aramă $\mathrm{V}$, Evaluation of bone mineral density and correlations with inflammation markers in romanian HIV-positive patients undergoing combined antiretroviral therapy. Farmacia, 2017; 65(1): 114-119.

18. Keys A, Fidanza F, Karvonen MJ, Kimura N, Taylor $\mathrm{HL}$, Indices of relative weight and obesity. Int $J$ Epidemiol., 2014; 43(3): 655-665.

19. Thacher TD, Clarke BL, Vitamin D insufficiency. Mayo Clin Proc., 2011; 86(1): 50-60.

20. Shepherd JA, Schousboe JT, Broy SB, Engelke K, Leslie WD, Executive summary of the 2015 ISCD Position development conference on advanced measures from DXA and QCT: Fracture prediction beyond BMD. J Clin Densitom., 2015; 18(3): 274-286.

21. McGettrick P, Barco EA, Mallon PWG, Ageing with HIV. Healthcare (Basel), 2018; 6(1): 1-19.

22. National Commission for Fighting Anti-AIDS statistical data, available in Romanian, at: http://cnlas.ro.

23. Cervero M, Torres R, Agud JL, Alcázar V, Jusdado JJ, García-Lacalle C, Moreno S, Prevalence of and risk factors for low bone mineral density in Spanish treated HIV-infected patients. PloS One, 2018; 13(4): 1-18.

24. Asomaning K, Bertone-Johnson ER, Nasca PC, Hooven F, Pekow PS, The association between body mass index and osteoporosis in patients referred for a bone mineral density examination. $J$ Womens Health (Larchmt)., 2006; 15(9): 1028-1034.

25. Pinnetti C, Federico L, Lorenzini P, Domenico C, Rita B, Laura L, Zaccarelli M, Cicalini S, Libertone R, Mosti S, Rizzi EB, Antinori A, Ammassari A, Relationship between body mass index and bone mineral density in HIV-infected patients referred for DXA. J Int AIDS Soc., 2014; 17(4 Suppl 3): 1.

26. Grigorie D, Coles D, Caragheorgheopol A, Șucaliuc A, Vitamin D status and consequences of long term supplementation with oral native vitamin D3 on the severity of primary hyperparathyroidism - the Romanian experience. Farmacia, 2018; 66(5): 877882.

27. Camargo MBR, Kunii LS, Hayashi LF, Muszkat $P$, Anelli CG, Marin-Mio RV, Martini LA, França N, Lazaretti-Castro M, Modifiable factors of vitamin D status among a Brazilian osteoporotic population 
attended a public outpatient clinic. Arq Bras Endocrin Metabol., 2014; 58(5): 572-582.

28. Viard JP, Souberbielle JC, Kirk O, Reekie J, Knysz B, Losso M, Gatell J, Pedersen C, Bogner JR, Mocroft A, EuroSIDA Study Group, Vitamin D and clinical disease progression in HIV infection: results from the EuroSIDA study. AIDS, 2011; 25(10): 1305-1315.

29. Li Vecchi V, Soresi M, Giannitrapani L, Mazzola G, La Sala S, Tramuto F, Caruso G, Colomba C, Mansueto P, Madonia S, Montalto G, Di Carlo P, Dairy calcium intake and lifestyle risk factors for bone loss in HIV-infected and uninfected Mediterranean subjects. BMC Infect Dis., 2012;12: 1-10.

30. Theodorou M, Sersté T, Van Gossum M, Dewit S, Factors associated with vitamin D deficiency in a population of $2044 \mathrm{HIV}$-infected patients. Clin Nutr., 2014; 33(2): 274-279.

31. Wohl DA, Orkin C, Doroana M, Pilotto JH, Yeni P, Vanveggel S, Deckx H, Boven K, Change in vitamin $\mathrm{D}$ levels and risk of severe vitamin D deficiency over 48 weeks among HIV-1-infected, treatment-naive adults receiving rilpivirine or efavirenz in a Phase III trial (ECHO). Antivir Ther., 2014; 19(2): 191-200.

32. Yin M, Vitamin D, bone, and HIV infection. Top Antivir Med., 2012; 20(5): 168-172.

33. Dao CN, Patel P, Overton ET, Rhame F, Pals SL, Johnson C, Bush T, Brooks JT; Study to Understand the Natural History of HIV and AIDS in the Era of Effective Therapy (SUN) Investigators, Low vitamin D among HIV-infected adults: prevalence of and risk factors for low vitamin D Levels in a cohort of HIVinfected adults and comparison to prevalence among adults in the US general population. Clin Infect Dis., 2011; 52(3): 396-405.

34. Mueller NJ, Fux CA, Ledergerber B, Elzi L, Schmid P, Dang T, Magenta L, Calmy A, Vergopoulos A, Bischoff-Ferrari HA; Swiss HIV Cohort Study, High prevalence of severe vitamin D deficiency in combined antiretroviral therapy-naive and successfully treated Swiss HIV patients. AIDS, 2010; 24(8): 1127-1134.

35. Atteritano M, Mirarchi L, Venanzi-Rullo E, Santoro D, Iaria C, Catalano A, Lasco A, Arcoraci V, Lo Gullo A, Bitto A, Squadrito F, Cascio A, Vitamin D status and the relationship with bone fragility fractures in HIV-infected patients: A case control study. Int $J$ Mol Sci., 2018; 19(1): 1-9.

36. Fakruddin JM, Laurence J, Interactions among human immunodeficiency virus (HIV)-1, interferon-gamma and receptor of activated NF-kappa B ligand (RANKL): implications for HIV pathogenesis. Clin Exp Immunol., 2004; 137(3): 538-545.

37. Gibellini D, Borderi M, De Crignis E, Cicola R, Vescini F, Caudarella R, Chiodo F, Re MC, RANKL/ OPG/TRAIL plasma levels and bone mass loss evaluation in antiretroviral naive HIV-1-positive men. J Med Virol., 2007; 79(10): 1446-1454.

38. Gazzola L, Bellistri GM, Tincati C, Ierardi V, Savoldi A, Del Sole A, Tagliabue L, d'Arminio Monforte A, Marchetti G, Association between peripheral TLymphocyte activation and impaired bone mineral density in HIV-infected patients. J Transl Med., 2013; 11: 1-10.

39. Aydın OA, Karaosmanoglu HK, Karahasanoglu R, Tahmaz M, Nazlican O, Prevalence and risk factors of osteopenia/osteoporosis in Turkish HIV/AIDS patients. Braz J Infect Dis., 2013; 17(6): 707-711.

40. Bedimo R, Maalouf NM, Zhang S, Drechsler H, Tebas P, Osteoporotic fracture risk associated with cumulative exposure to tenofovir and other antiretroviral agents. AIDS, 2012; 26(7): 825-831.

41. Horizon AA, Joseph RJ, Liao Q, Ross ST, Pakes GE, Characteristics of foot fractures in HIV-infected patients previously treated with tenofovir versus nontenofovir-containing highly active antiretroviral therapy. HIVAIDS (Auckl), 2011; 3: 53-59.

42. Stellbrink HJ, Orkin C, Arribas JR, Compston J, Gerstoft J, Van Wijngaerden E, Lazzarin A, Rizzardini G, Sprenger HG, Lambert J, Sture G, Leather D, Hughes S, Zucchi P, Pearce H; ASSERT Study Group, Comparison of changes in bone density and turnover with abacavir-lamivudine versus tenofovir-emtricitabine in HIV-infected adults: 48-week results from the ASSERT study. Clin Infect Dis., 2010; 51(8): 963-972.

43. Gutiérrez F, Masiá M, The role of HIV and antiretroviral therapy in bone disease. AIDS Rev., 2011; 13(2): 109-118. 